\title{
SOP Hämodynamisches Monitoring
}

Björn Ellger, Julian Bösel, Tobias Schürholz

\section{Einleitung zur Rubrik}

In dieser Rubrik stellen wir Standard Operating Procedures (SOPs) für häufige, intensivmedizinisch relevante Prozesse vor. Die Form ist eher im Sinne einer Schablone zu verstehen als - durchaus subjektiv gefärbte Anregung, eigene, auf lokale Gegebenheiten adaptierte stationsinterne SOPs zu entwerfen und zu implementieren.

\section{Einleitung zur SOP Hämodynamisches Monitoring}

Evidenz-basierte Empfehlungen für den Einsatz des hämodynamischen Monitorings existieren derzeit nur für den Bereich der herzchirurgischen Intensivmedizin [1]. Für alle anderen Patienten stützt sich die vorliegende SOP im Wesentlichen auf Prinzipien der „good clinical practice“. Dies liegt vor allem an der schwachen Evidenz und macht das hämodynamische Monitoring zu einem sehr kontroversen Thema. Einige Parameter unterliegen sehr vielen Einflussfaktoren, sodass man sie immer mit Vorsicht interpretieren muss. Individuelle Erfahrungen und Sicherheit im Umgang mit einer Methode spielen eine große Rolle bei der Methodenauswahl. Immerhin mit guter Evidenz abgesichert ist inzwischen, dass der ZVD als Monitoringparameter für den intravaskulären Volumenbedarf des Intensivpatienten unbrauchbar ist [2].

Eine individuelle Anpassung der Therapie an die Patientenbedürfnisse und die sich ändernde Krankheitssituation sowie individuelle Gegebenheiten des Patienten (z.B. nicht kanülierbare Arterien, Kontra- indikationen für TEE, schlechte Schallbedingungen im TTE) ist notwendig. Generell gilt: so wenig und so wenig invasiv wie möglich, so viel und so invasiv wie nötig. Nichtinvasive Verfahren (z. B. EKG, Pulsoximetrie) sind in der Regel zuverlässig, kostengünstig und schnell verfügbar, was von vielen invasiven Verfahren nicht behauptet werden kann. Vor allem die invasiven Verfahren haben immer Komplikationsmöglichkeiten (z.B. Ösophagusverletzungen bei der Transösophagealen Echokardiografie (TEE), Infektionen beim ZVK, Klappenverletzungen oder Rhythmusstörungen beim pulmonalarteriellen Katheter (PAK)). Gemäß individueller Einschätzung kann das Monitoring um z. B. nichtinvasives HZV-Monitoring ergänzt werden. Evidenz, die ein solches Vorgehen unterstützt oder kategorisch ablehnt, gibt es zur Zeit allerdings so gut wie nicht [3]. Angesichts dessen sollte man nicht versuchen, den Volumenstatus bzw. die Kreislauffunktion des Intensivpatienten anhand nur eines Messwerts zu bestimmen, sondern mehrere Parameter integrieren und gleichzeitig auch auf Plausibilität prüfen. Statt Absolutwerten scheint sich zunehmend die Parameterveränderung auf Volumengabe bzw. -verschiebung durchzusetzen. Man sollte sich immer klar machen, dass nicht valide Messwerte, sei es durch unzureichende Methodik, schlechte Messdurchführung oder Fehlinterpretation, zu falschen Therapieentscheidungen führen können („A fool with a tool is still a fool“). Zur Zeit erarbeitet die Deutsche Gesellschaft für Anästhesiologie und Intensivmedizin (DGAI) eine S-3-Leitlinie zum hämodynamischen Monitoring, die die einzelnen Methoden in den Zusammenhängen sicher sinnvoll einordnen und klare Empfehlungen aussprechen wird.

Alle bisher publizierten SOPs der Intensivmedizin up2date finden Sie online unter www.thieme.de/ intensiv-u2d/sops.
Wenn Sie selbst in Ihrer Klinik eine SOP haben, die auch anderen Kollegen nützlich sein könnte, freuen wir uns über eine kurze E-Mail an intensiv-u2d@thieme.de. 


\section{SOP Hämodynamisches Monitoring}

Aufnahme kritisch kranker Patient auf ICU

Etablieren Basismonitoring'

- EKG: kontinuierlich II und V5 (alternativ II und V3, II und V4 oder V3, 4, 5),

ST-Streckenanalyse, 12-Kanal (archivieren!) immer bei Aufnahme, bei

herzchirurgischen Patienten zusätzlich nach 6 Stunden, bei Myokardinfarkt zusätzlich

nach 3 Stunden

- Pulsoxymetrie

- Nichtinvasive Blutdruckmessung

- Kapnografie/-metrie (bei beatmeten Patienten)

- Temperaturmessung (kontinuierlich, aber mindestens 4-stündlich)

- Bilanzierung (In den ersten 24 Stunden stündlich, danach in 4-stündlichen Intervallen)

- Blutgasanalyse (BGA) bei Aufnahme (Wiederh. innerhalb 30 min bei hämodyn. Insta-

bilität oder Veränderung der Ventilation, danach alle $6 \mathrm{~h}$ oder auf spezielle Indikation)

- Wenn ZVK vorhanden $\mathrm{S}_{\mathrm{cv}} \mathrm{O}_{2}$ (Wiederholung alle $12 \mathrm{~h}$ oder nach Therapiemodifikation)

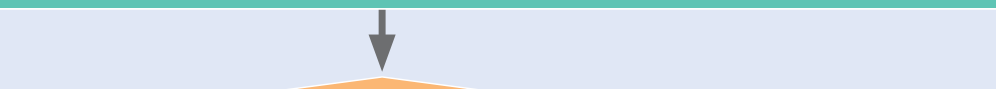

Hämodynamische Instabilität?2

nein

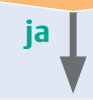

- Invasive arterielle Blutdruckmessung, wenn noch nicht vorhanden ${ }^{3}$

- "Passive legraising“ (PLR): $\triangle$-MAP > $10 \%$ = Volumenbedarf (CAVE: Hirnläsion,

Rechtsherzversagen $)^{4}$

- Anlage ZVK und Messung der $\mathrm{S}_{\mathrm{cV}} \mathrm{O}_{2}^{5}$

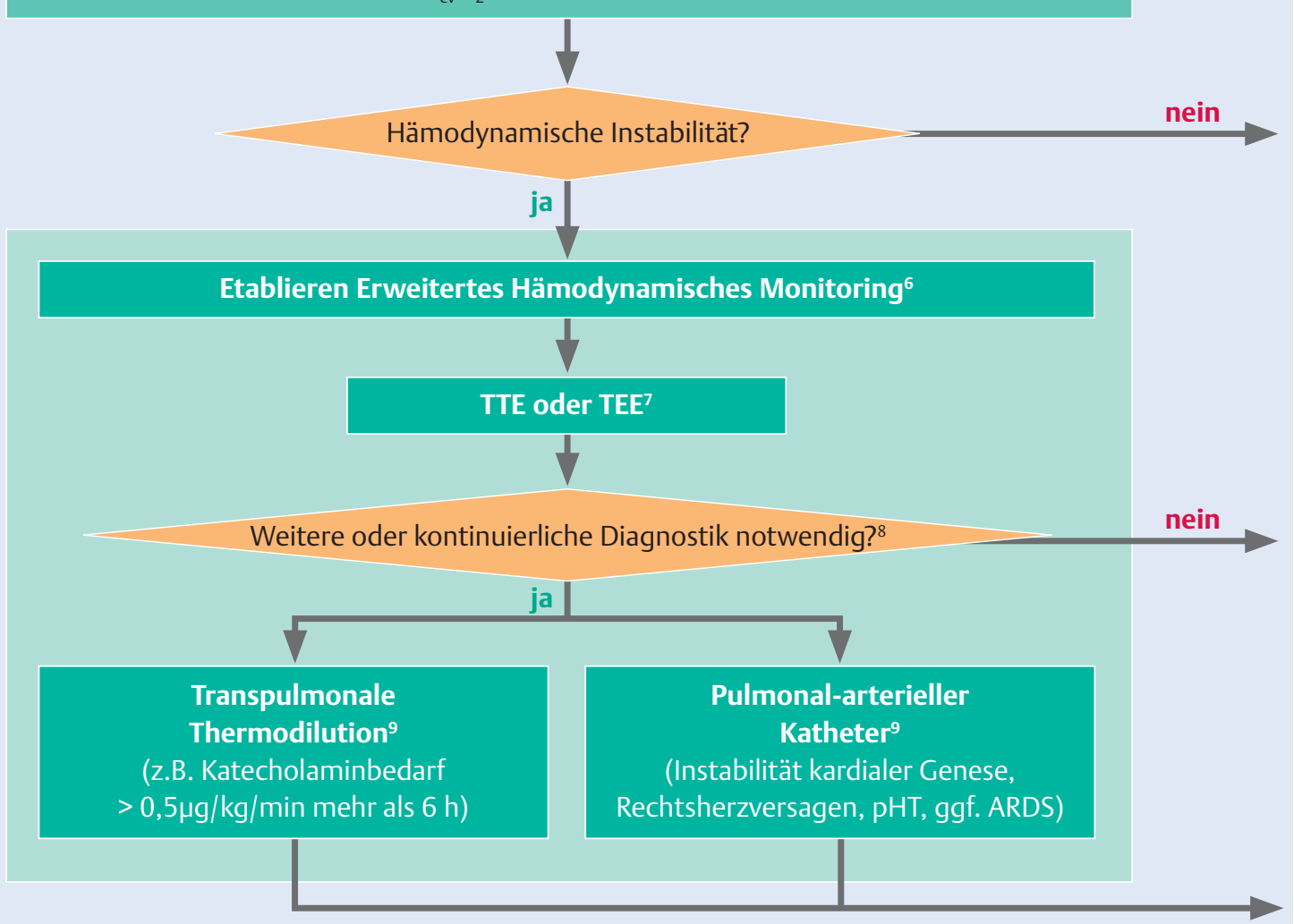




\section{Erläuterungen zur SOP Hämodynamisches Monitoring}

1. Das Basismonitoring ist wenig invasiv und dient dazu, eine drohende/manifeste hämodynamische Instabilität zu erkennen. Es ist bei allen kritisch kranken Patienten ein obligater Bestandteil der Überwachung. Messintervalle können zunächst vorgegeben werden, müssen dann aber individuell angepasst werden. Auch scheinbar banale Dinge wie Bilanz oder Gewichtsverlauf gehören zum Basismonitoring. Die Registrierung eines 12-Kanal EKGs sollte bei jedem Patienten bei Aufnahme zusätzlich zum kontinuierlichen EKG-Monitoring erfolgen.

2. Die Definition der hämodynamischen Instabilität ist schwierig und wieder individuell für jeden einzelnen Patienten zu definieren. Auch Zielparameter der Hämodynamiktherapie sollten individualisiert festgelegt werden. Zeichen der mangelhaften Mikroperfusion wie Laktatanstieg, niedrige gemischtvenöse Sättigung, akutes Nierenversagen oder eine schlechte Rekapillarisierungszeit sind genauso relevant wie ein inadäquater Blutdruck (Hyper- und Hypotonie), Arrhythmien, Dyspnoe, Störungen des Gasaustauschs oder eine Veränderung des mentalen Status.

3. Invasive arterielle Blutdruckmessung: Die arterielle Kanülierung ist wenig invasiv und erlaubt eine kontinuierliche Überwachung des systemarteriellen Druckes. Die Komplikationsraten sind gering, daher kann man die Indikation großzügig stellen. Indikationen:

- Hämodynamisch instabile Patienten (z. B. Patienten mit manifester/drohender Katecholaminabhängigkeit ab $0,1 \mu \mathrm{g} / \mathrm{Kg} / \mathrm{min}$ )

- Patienten mit respiratorischer Insuffizienz und Beatmungsbedarf

- Patienten mit bedrohter Organfunktion

(z.B. Hirnläsion)

4. PLR (passive leg raising): In Abwesenheit von Kontraindikationen (z. B. Rechtsherzversagen) stellt das passive Anheben beider Beine des Patienten eine exzellente Möglichkeit dar, einen intravasalen Volumenmangel zu erkennen und die Reagibilität der Kreislaufverhältnisse auf Volumengabe reversibel zu testen. Bei einem Anstieg des arteriellen Mitteldrucks um mehr als $10 \%$ bei PLR kann von einem intravasalen Volumenmangel ausgegangen werden und der Patient profitiert mit recht hoher Wahrscheinlichkeit von einer Volumengabe.

5. Venöse Oximetrie: Die zentralnervöse $\mathrm{O}_{2}$-Sättigung $\left(\mathrm{S}_{\mathrm{cv}} \mathrm{O}_{2}\right)$ gilt als Surrogat für ein ausreichendes Herzzeitvolumen. Aber auch bei maldistributivem Schock oder anderen Sauerstoffverwertungsstörungen kann

\section{Tabelle 1}

Gegenüberstellung der wichtigsten Spezifika einzelner Monitoringverfahren

\begin{tabular}{|c|c|c|c|}
\hline & PAK & PICCO/EV-1000 & FloTrac/Vigileo \\
\hline $\begin{array}{l}\text { HZV- } \\
\text { Messung }\end{array}$ & $\begin{array}{l}\text { Pulmonalarterielle } \\
\text { Thermodilution: } \\
\text { kontinuierlich vs. } \\
\text { intermittierend }\end{array}$ & $\begin{array}{l}\text { Transpulmonale } \\
\text { Thermodilution + } \\
\text { Pulskonturanalyse }\end{array}$ & $\begin{array}{l}\text { Unkalibrierte } \\
\text { Pulskonturanalyse }\end{array}$ \\
\hline Vorlast & $\begin{array}{l}\text { ZVD, PAOP: } \\
\text { Bilden die kardiale Vor- } \\
\text { last nur ungenügend ab, } \\
\text { sind aber wichtige } \\
\text { Verlaufsparameter zur } \\
\text { Abschätzung der ventri- } \\
\text { kulären Compliance und } \\
\text { des Füllungszustandes }\end{array}$ & $\begin{array}{l}\text { ITBI, GEDI: } \\
\text { Relativ genaue } \\
\text { Abschätzung der } \\
\text { kardialen Vorlast }\end{array}$ & - \\
\hline Nachlast & $\begin{array}{l}\text { SVR } \\
\text { PVR } \\
\text { Der PAK stellt die einzige } \\
\text { Möglichkeit dar, die RV- } \\
\text { Nachlast kontinuierlich } \\
\text { zu überwachen }\end{array}$ & SVR & SVR \\
\hline $\begin{array}{l}\text { Volumen- } \\
\text { reagibilität }\end{array}$ & & SVV/PPV & SVV \\
\hline $\begin{array}{l}\text { Andere } \\
\text { Parameter }\end{array}$ & $\begin{array}{l}\mathrm{S}_{\mathrm{cv}} \mathrm{O}_{2} \\
\text { Sauerstoffstatus: } \\
\mathrm{DO}_{2} / \mathrm{VO}_{2}\end{array}$ & EVLW & - \\
\hline Invasivität & Hoch & Mittel & Niedrig \\
\hline Nachteile & $\begin{array}{l}\text { Hohe Invasivität und } \\
\text { Komplikationen (z. B. } \\
\text { Infektionen, Arryth- } \\
\text { mien, Klappenschäden) }\end{array}$ & $\begin{array}{l}\text { Spezieller Katheter } \\
\text { erforderlich, femo- } \\
\text { rale/brachiale Punk- } \\
\text { tion erforderlich }\end{array}$ & $\begin{array}{l}\text { Außer HZV wenig } \\
\text { zusätzliche Para- } \\
\text { meter } \\
\text { Sehr schlecht } \\
\text { validiert }\end{array}$ \\
\hline Vorteile & $\begin{array}{l}\text { Klinischer Goldstandard } \\
\text { der HZV-Messung, } \\
\text { umfassender hämo- } \\
\text { dynamischer Status, } \\
\text { hoher edukativer Wert }\end{array}$ & Volumetrie & $\begin{array}{l}\text { Kann mit jedem } \\
\text { Katheter in jeder } \\
\text { Arterie messen } \\
\text { „Plug and Play“ }\end{array}$ \\
\hline
\end{tabular}

die $\mathrm{S}_{\mathrm{cv}} \mathrm{O}_{2}$ normal oder sogar erhöht sein. Eine niedrige $\mathrm{S}_{\mathrm{cv}} \mathrm{O}_{2}$ kann bei strukturell bedingter Herzinsuffizienz (z.B. Kardiomyopathie, Ischämie) für den Patienten normal sein und Therapiebemühungen resultieren dann nicht in einer Verbesserung der Hämodynamik, sondern lediglich in unerwünschten Ereignissen. Besondere Indikationen sind:

- Patienten in der Initialphase des septischen

Schocks (innerhalb der ersten $6 \mathrm{~h}$ ): zumindest alle

$3 \mathrm{~h}$ intermittierende Bestimmung der $\mathrm{S}_{\mathrm{cv}} \mathrm{O}_{2}$

- Postoperative herzchirurgische Patienten: bei Aufnahme, nach 4 und $12 \mathrm{~h}$ 
- Akut insbesondere bei neu aufgetretener hämodynamischer Instabilität oder nicht stabilisierbarem Schock

6. Das erweiterte hämodynamische Monitoring dient sowohl dazu, die einer hämodynamischen Instabilität zugrunde liegende Ursache zu erkennen, als auch die hämodynamische Therapie rational zu steuern. Gängige Verfahren sind: Echokardiografie (transthorakal (TTE) oder transösophageal (TEE)), Pulmonalarterienkatheter (PAK), transpulmonale Thermodilution + kalibrierte Pulskonturanalyse (PICCO/EV1000), unkalibrierte Pulskonturanalyse (FloTrac/ Vigileo).

7. Echokardiografische transösophageale Untersuchungen sollen nur von in der Methode ausgebildeten und erfahrenen Untersuchern durchgeführt werden. Grundsätzlich sollte jeder Patient mit hämodynamischer Instabilität zumindest einmal eine Echokardiografie erhalten zur Detektion von strukturellen Herzerkrankungen (z.B. Vitien) und anderen behebbaren Ursachen (z. B. Perikardtamponade) [4].

8. Differenzialindikationen zum Einsatz des erweiterten hämodynamischen Monitorings. Generell sind diejenigen Verfahren bevorzugt einzusetzen, mit denen der Anwender sich gut auskennt.

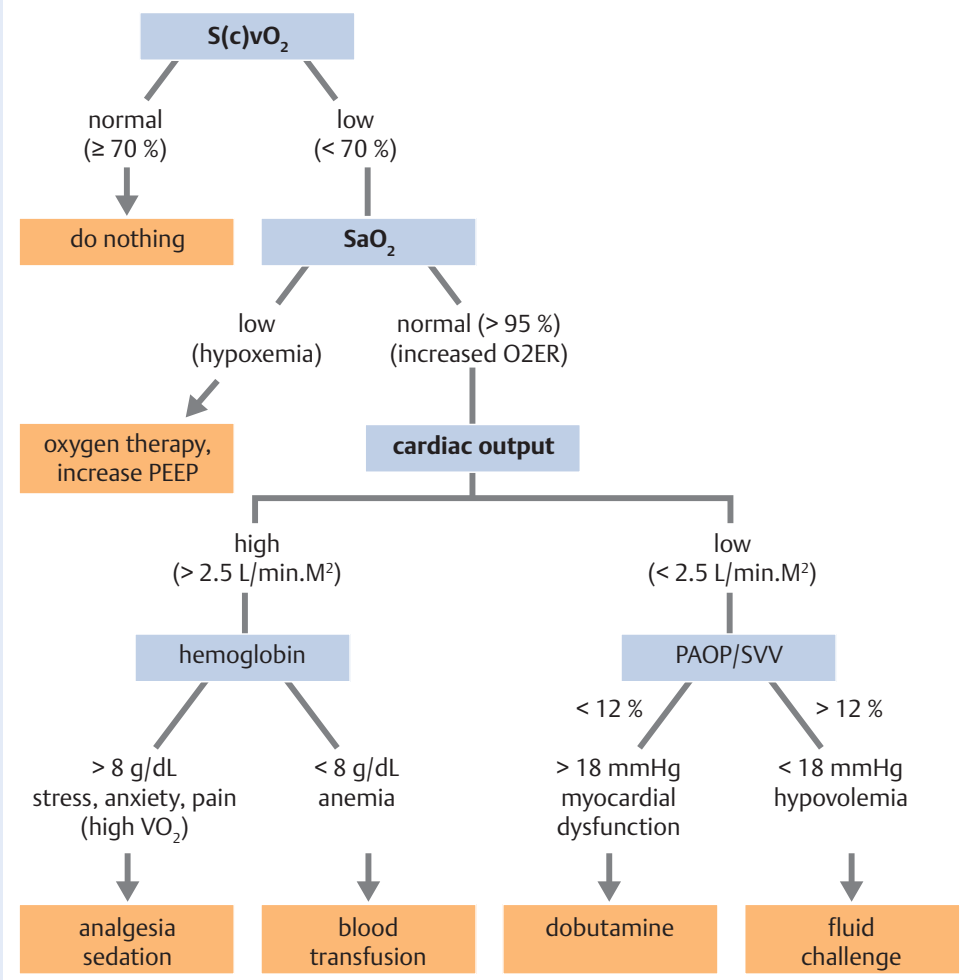

Nach: Pinsky M, Vincent J-L: Let us use the pulmonary artery catheter correctly and only when we need it. Crit Care Med 2005; 331119 - 1122 [6].
- TEE/TTE (Methodik der ersten Wahl, jeder Patient mit hämodynamischer Instabilität braucht eine Ultraschalluntersuchung):

- hämodynamische Instabilität unklarer/kardialer Genese

- (Klappen)Vitien

- (regionale/globale) systolische und diastolische Pumpfunktion von beiden Ventrikeln

- Endokarditisverdacht

- Ausschluss intrakavitärer Thromben (TEE)

- PAK [5] (vor allem wenn kontinuierliche Aussagen über das pulmonale Strombett benötigt werden): - hämodynamische Instabilität kardialer Genese

- Rechtsherzversagen

- schwerer pulmonaler Hypertonus

- ggf. ARDS

- ggf. Patienten, bei denen inhalierte Vasodilatatoren zum Einsatz kommen (inhalatives NO, inhaliertes Iloprost)

- PICCO/EV-1000 (vor allem, wenn ein kontinuierliches Monitoring über einen längeren Zeitraum gebraucht wird):

- Patienten mit anhaltend hohem Katecholaminbedarf für $>6$ Stunden (z. B. Noradrenalin oder Adrenalin $>0,5 \mu \mathrm{g} / \mathrm{kg} / \mathrm{min}$ ) trotz adäquater Volumentherapie

- Hämodynamisch instabile Patienten (wenn genaue Abschätzung von Vorlast und EVLW wichtig erscheint)

- Patienten, bei denen die Ursache für die hämodynamische Instabilität nicht offensichtlich ist

- ggf. ARDS

- ggf. Patienten, bei denen inhalierte Vasodilatatoren zum Einsatz kommen (inhalatives NO, inhaliertes Iloprost)

- Kontraindikationen/Nutzungseinschränkungen zum Einsatz des erweiterten hämodynamischen Monitorings

- TTE: keine, kann aber technisch schwierig sein (z.B. kritische Physiognomie, Emphysem)

- TEE:

- absolut: Tumoren des oberen Gastrointestinaltrakts, Ösophagusruptur, bekannte Strikturen

- relative: Malformationen, Blutungen, Varizen und Zustand nach chirurgischen Eingriffen im Bereich des oberen Gastrointestinaltrakts - PAK:

- absolut: Rechtsherzendokarditis, Thromben im rechten Herzen, mechanische Trikuspidaloder Pulmonalklappe

- Eingeschränkter Nutzen bei relevanten Klappeninsuffizienzen 
- PICCO/EV-1000: Einschränkungen für die Nutzung: arterielle Verschlusskrankheit, signifikante Klappeninsuffizienzen, intraarterielle Gegenpulsation oder extrakorporale Zirkulation, SVV ist nur unter kontrollierter mechanischer Ventilation und bei Sinusrhythmus verwertbar

9. Vergleich der Verfahren des katheterbasierten erweiterten hämodynamischen Monitorings. Da es sinnvoll ist, individuelle Trigger für die Erweiterung des Monitorings festzulegen, kann das Erreichen einer bestimmten Katecholamindosis (z. B. 0,5 $\mu$ g/ $\mathrm{kg} / \mathrm{min}$ ) oder eine bestimmte zugeführte Volumenmenge als Trigger definiert werden. Generell muss man sagen, dass es kaum Studien gibt, die einen Vorteil des erweiterten Monitorings gegenüber einer Standardtherapie zeigen. Die Diskussion hierüber ist aber nicht Gegenstand dieser SOP. In den Händen des Geübten bieten aber die Verfahren wertvolle Zusatzinformationen über den Patienten, die in das Gesamtbild integriert werden müssen.

10. Aus dem hämodynamischen Monitoring muss eine Therapie erwachsen. Hier gibt es publizierte Vorschläge, die in dieser SOP aber nicht weiter vertieft werden sollen. Ein gut publiziertes Beispiel unter Einbeziehung des PAK sei hier einmal exemplarisch wiedergegeben [6]. Es sei angemerkt, dass über jeden der zur Entscheidung angegebenen Absolutwerte, insbesondere der Transfusionstrigger, vortrefflich gestritten werden kann.

\section{Abkürzungen}

$\mathrm{DO}_{2}$

GEDI

ITBI

EVLW

HZV

PAOP

PAK

pHT

PLR

PPV

PVR

$\mathrm{S}_{\mathrm{cv}} \mathrm{O}_{2}$

$\mathrm{S}_{\mathrm{a}} \mathrm{O}_{2}$

SVR

SVV

TEE

TTE

$\mathrm{VO}_{2}$

ZVD

\section{Sauerstoffangebot} globales enddiastolisches Volumen (Index) intrathorakales Blutvolumen (Index) extravaskuläres Lungenwasser Herzzeitvolumen pulmonalarterieller Okklusionsdruck pulmonalarterieller Katheter pulmonalarterielle Hypertonie passive leg raising Pulsdruckvariation pulmonalvaskulärer Widerstand zentralvenöse Sauerstoffsättigung arterielle Sauerstoffsättigung systemvaskulärer Wiederstand Schlagvolumenvarianz transösophageale Echokardiografie transthorakale Echokardiografie Sauerstoffverbrauch zentralvenöser Druck

\section{Über die Autoren}

\section{Björn Ellger}

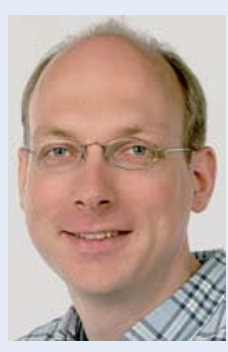

Prof. Dr. med. Björn Ellger ist Leiter der operativen Intensivstationen des Universitätsklinikums Münster. E-Mail: ellger@anit.uni-muenster.de

Julian Bösel

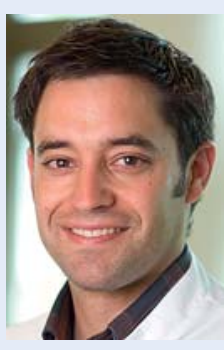

PD Dr. med. Julian Bösel ist Oberarzt der neurologischen Intensivstation des Universitätsklinikums Heidelberg. E-Mail: julian.boesel@med.uniheidelberg.de

Tobias Schürholz

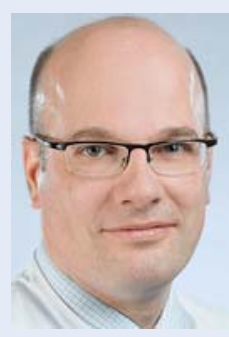

PD Dr. med. Tobias Schürholz ist leitender Oberarzt der Klinik für Operative Intensivmedizin und Intermediate Care des Universitätsklinikums Aachen.

E-Mail: tschuerholz@ukaachen.de

\section{Weiterführende Literatur}

1 Leitlinie DGAI/AWMF: Intensivmedizinische Versorgung herzchirurgischer Patienten - Hämodynamisches Monitoring und Herz-Kreislauf.http://www.awmf.org/leitlinien/detail/I/001016.html

2 Leitlinie: DGAI/ AWMF. Intravasale Volumentherapie beim Erwachsenen.http://www.awmf.org/leitlinien/detail/II/001020.html

3 Vincent JL, Pelosi P, Pearse R et al. Perioperative cardiovascular monitoring of high-risk patients: a consensus of 12 . Crit Care 2015; 19: 224; DOI 10.1186/s13054-015-0932-7

4 Treskatsch S, Habicher M, Sander M. Echocardiography for hemodynamic monitoring on ICU? Anasthesiol Intensivmed Notfallmed Schmerzther 2014; 49: 708-717

5 Schürholz T, Marx G. Is there any indication for perioperative use of the pulmonary artery catheter? Anasthesiol Intensivmed Notfallmed Schmerzther 2012; 47: 110-117

6 Pinsky M, Vincent J-L. Let us use the pulmonary artery catheter correctly and only when we need it. Crit Care Med 2005; 33: $1119-1122$ 